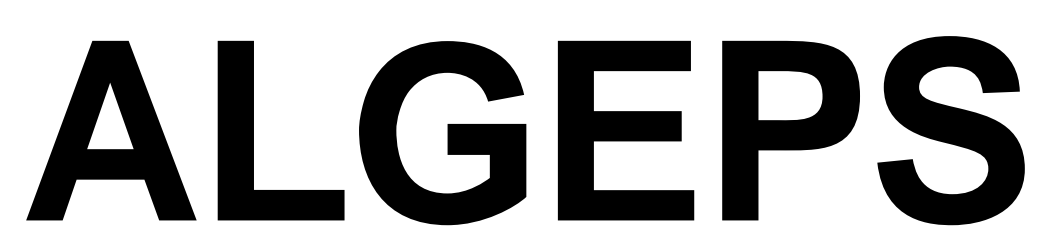

REVISTA DE GEOLOGIA, SÈRIE B no 581 - Febrer del 2012

ISSN $1132-7014$

D.L.B. 28.178 - 92

12 pàgines

\begin{abstract}
RECORRIDO DESDE TARAZONA A LOS FAYOS, SANTA CRUZ DEL MONCAYO Y AL SANTUARIO DEL MONCAYO, A TRAVÉS DEL PATRIMONIO GEOLÓGICO Y MINERO DE LA COMARCA DEL MONCAYO
\end{abstract}

Josep M. Mata-Perelló

Aquest recorregut va ésser experimentat amb docents el dia 2 DE JUNY DEL 2010 


\section{RECORRIDO DESDE TARAZONA A LOS FAYOS, SANTA CRUZ DEL MONCAYO Y AL SANTUARIO DEL MONCAYO, A TRAVÉS DEL PATRIMONIO GEOLÓGICO Y MINERO DE LA COMARCA DEL MONCAYO}

\section{ADVERTENCIAS PREVIAS}

Como en otros recorridos de RECONOCIMIENTO GEOLÓGICO (o de RECONOCIMIENTO GEOLÓGICO Y MINERO), el recorrido se compondrá de diversas PARADAS. En este caso serán seis.

Por otra parte, habrá que tener en cuenta, en todo momento, especialmente antes de empezar los recorridos de los diferentes tramos, el estado de los caminos y carreteras, por donde transitará el recorrido. Precisamente, en este itinerario, se discurrirá en una pequeña parte del recorrido por caminos de tierra, entre Agramonte y el acceso al Santuario del Moncayo. Al respecto, cabe decir que prácticamente todo este tramo se halla en buenas condiciones.

Finalmente, como ya hacemos en otros recorridos similares, queremos decir que hace falta tener un cuidado muy especial en el respeto a la naturaleza, a lo largo de todo el recorrido del itinerario, y también fuera de él.

\section{BREVE INTRODUCCIÓN GEOLÓGICA}

El recorrido de este itinerario, se desarrollará exclusivamente por dos de las tres unidades geológicas que constituyen el suelo y el subsuelo de Aragón. Concretamente por la Depresión Geológica del Ebro (en donde se iniciará) y .por el Sistema Ibérico (en donde finalizará).

Así, a lo largo de todo de todo el recorrido del itinerario, se irán encontrando en un primer tramo (el desarrollado en la Depresión Geológica del Ebro) afloramientos cenozoicos, en su mayoría de carácter arcilloso y calcolutítico, pertenecientes generalmente al Mioceno.

Mientras que en el segundo tramo (el desarrollado por el Sistema Ibérico) afloramientos de los materiales mesozoicos (fundamentalmente del Triásico) y paleozoicos (del Carbonífero en su mayor parte).

Asimismo, a menudo, encontraremos afloramientos de los materiales detríticos cuaternarios, por encima de los anteriores 


\section{BREVE INTRODUCCIÓN GEOGRÁFICA}

El recorrido del presente itinerario se efectuará exclusivamente por una comarca aragonesa, por la del Moncayo (así, se transitara entre las inmediaciones de Tarazona y el fin del recorrido cerca del Santuario de la Virgen del Moncayo).

En este recorrido, se circulará por los términos municipales de: Tarazona, los Fayos, Santa Cruz del Moncayo y San Martín de la Virgen del Moncayo, todos ellos de la comarca del Moncayo

\section{OBJETIVOS GENERALES DE ESTE ITINERARIO}

En este itinerario, los objetivos generales que se han de conseguir, se pueden concretar en los siguientes aspectos:

1.- Estudio y reconocimiento de los materiales cenozoicos (en buena parte del Mioceno) situados en la Depresión Geológica del Ebro, que iremos encontrando a lo largo del recorrido del primer tramo del itinerario, entre Tarazona y las inmediaciones de San Martín de la Virgen del Moncayo.

2.- Estudio y reconocimiento de los materiales paleozoicos y mesozoicos situados en el Sistema Ibérico. Estos materiales los iremos encontrando a lo largo del segundo tramo el recorrido de este itinerario, especialmente entre Agramonte y el Santuario de la Virgen del Moncayo.

3.- Visión de algunas de las antiguas explotaciones encontradas a lo largo del recorrido del itinerario

4.- Visión de los diferentes lugares directamente relacionados con el Patrimonio Geológico y Minero que iremos encontrando a lo largo del recorrido del presente itinerario

\section{ANTECEDENTES BIBLIOGRÁFICOS}

En relación con este itinerario, no conocemos ningún antecedente, relativo a otro itinerario que discurra por este lugar. En este sentido, este itinerario constituye un antecedente, si no estamos equivocados.

Por otra parte, haremos mención de algunos trabajos, de carácter geológico generalista, que corresponden a los trabajos del IGME (1972, 1974 y 1975), relativos al Mapa Geológico de España (a Escala 1.200.000), al Mapa Metalogenético de España y al Mapa de Rocas Industriales de España

Con respecto a las mineralizaciones que iremos encontrando, mencionaremos los trabajos de: CALVO (2001), CALVO et altri (1988); MAESTRE (1845); así como nuestros trabajos: MATA-PERELLÓ (1987 y 2000). 
También mencionaremos el trabajo de PRAMES (2005) dedicado a la comarca del Bajo Martín. Así como el del GOBIERNO DE ARAGÓN (2001), dedicado a los Puntos de Interés Geológico de Aragón.

Finalmente, diremos que todos estos trabajos (así como otros que ahora no hemos aludido), figurarán mencionados, por orden alfabético, en el apartado dedicado a las REFERENCIAS BIBLIOGRÁFICAS.

\section{RECORRIDO DEL ITINERARIO}

Este recorrido se iniciará en las inmediaciones de Tarazona, dentro de la comarca del Moncayo (o del Somontano del Moncayo). Tras ello, el recorrido se dirigirá hacía los Fayos, en donde realizaremos una nueva parada.

Luego, el recorrido se dirigirá hacía el pueblo de Santa Cruz del Moncayo, en donde efectuaremos una parada, y otras dos en el vecino término de Tarazona. Tras ello, nos dirigiremos hacía el Santuario de la Virgen del Moncayo, en donde finalizará el recorrido, tras realizar algunas paradas más, dentro del término municipal de Tarazona.

\section{DESCRIPCIÓN DEL ITINERARIO}

Como de costumbre, haremos una serio de PARADAS (o ESTACIONES), en donde se realizaran diversas explicaciones en torno a las características del lugar en donde se halla la PARADA. Por otra parte, en ellas haremos mención del término municipal dónde se encuentran, así como del número del "Mapa Topográfico Nacional (a escala 1:50.000, que indicaremos entre paréntesis. Así, ahora (en este recorrido) utilizaremos solamente dos hojas: concretamente la $\mathbf{3 2 0}$ (o de Tarazona) y la $\mathbf{3 5 2}$ (o de Tabuenca).

Así, la relación ordenada de las paradas que constituyen el recorrido de este itinerario, es la siguiente:

PARADA 1. FÁBRICA DE CERAMICA DE TARAZONA, (término municipal de Tarazona, comarca del Moncayo). (Hoja 320).

El recorrido del presente itinerario conviene iniciarlo en la localidad de Tarazona, la capital de la comarca. Concretamente, puede iniciarse en la carretera que conduce a Santa Cruz de Moncayo. En esta carretera, casi a la salida de Tarazona, realizaremos una parada en la antigua fábrica de cerámica. Ésta se halla situada a la derecha de la carretera, saliendo.

En este breve recorrido (apenas $1 \mathrm{Km}$ ) habremos visto afloramientos de los materiales cenozoicos que rellenan la Depresión Geológica del Ebro. En esta zona, los 
materiales son muy detríticos. Por otra parte, por encima de estos materiales cenozoicos del Mioceno, se observan depósitos de terrenos cuaternarios.

En este lugar había una antigua fábrica de cerámica, en la que se utilizaban materiales lutíticos miocénicos. En la actualidad la fábrica está cerrada y en un creciente estado de deterioro.

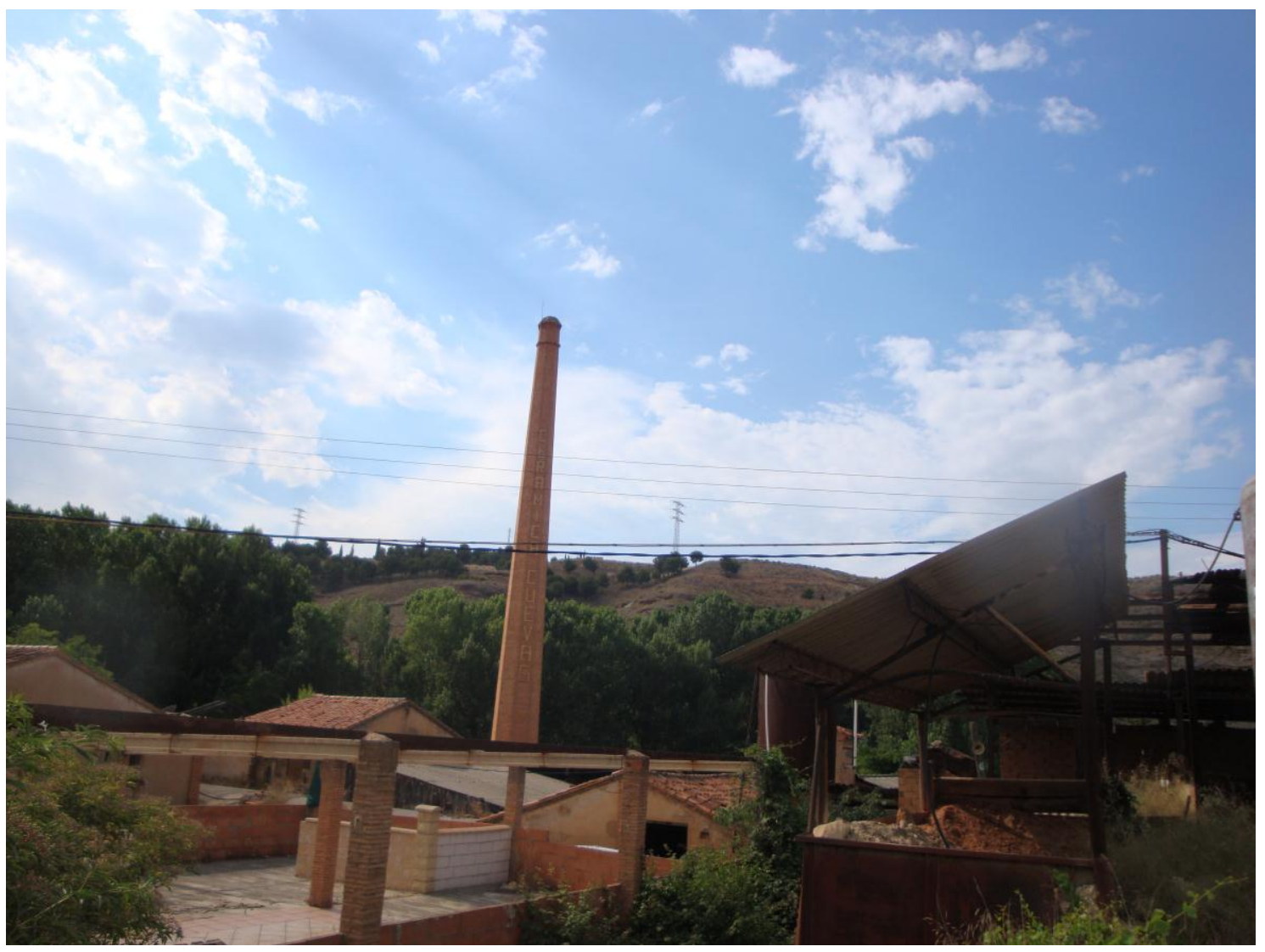

Antigua fábrica de cerámica de Tarazona

PARADA 2. LOS MALLOS DE LOS FAYOS, (término municipal de los Fayos, comarca del Moncayo). (Hoja 320).

Después de realizar la parada anterior, volver a la carretera N-211, con la finalidad de ir hacia el poniente. Al llegar al cruce de Torrellas, nos convendrá tomarlo con la finalidad de ir después hacía los Fayos. Al llegar a ese pueblo, nos convendrá seguir por la carretera que remonta el río Queiles. A poco más de 1'5 Km de los Fayos, realizaremos una nueva parada. Así, desde la anterior, habremos recorrido unos $8 \mathrm{Km}$, para llegar hasta aquí.

En este recorrido, nos habremos desplazado siempre entre los afloramientos de los materiales cenozoicos (del Mioceno) y cuaternarios que rellenan la Depresión Geológica del Ebro, en donde ahora continuamos estando situados.

En este lugar se hacen patentes (mirando hacia el poniente) unos impresionantes relieves de conglomerados. Estos presentan un buzamiento horizontal y son claramente 
postorogénicos. En este lugar, estos materiales dan lugar a unas impresionantes formas de relieve que hemos denominado como los Mallos de los Fayos. Por otra parte, se trata de conglomerados carbonatados sobre los que se han desarrollados buenos ejemplos de karstificación.

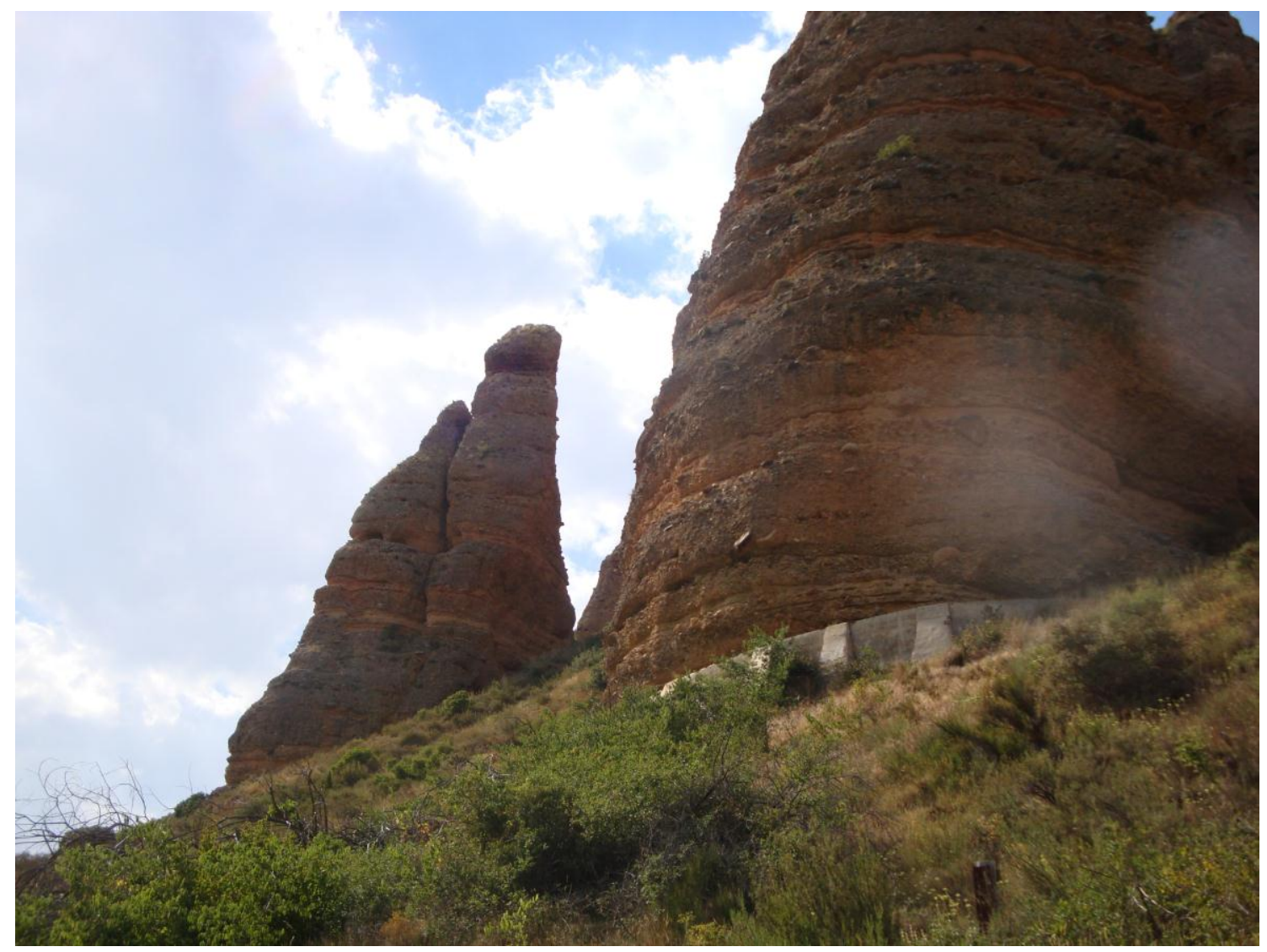

Los Mallos de los Fayos

PARADA 3. TEJAR DE SANTA CRUZ DE MONCAYO, (término municipal de Santa Cruz del Moncayo, comarca del Moncayo). (Hoja 320).

Después de realizar la parada anterior, conviene continuar ahora por la carretera que se dirige hacia las cercanías de Santa Cruz de Moncayo. Al llegar al cruce de carreteras, nos dirigiremos hacia este último pueblo mencionado. A menos de $1^{\prime} 2 \mathrm{Km}$ antes de llegar, efectuaremos una nueva parada, a unos $5 \mathrm{Km}$ (aproximadamente) de la parada anterior.

En este recorrido habremos ido encontrando afloramientos de los materiales cenozoicos mencionados en las paradas anteriores. Estos materiales cenozoicos del Mioceno rellenan en este sector la Depresión Geológica del Ebro, en donde estamos ahora situados.

En este lugar hay unos afloramientos de los materiales lutíticos cenozoicos, pertenecientes al Mioceno. Estos materiales de color rojizo - marronoso, han sido 
explotados en este lugar para ser utilizados como materia prima en una tejera. Ésta se halla en un avanzado estado de deterioro.

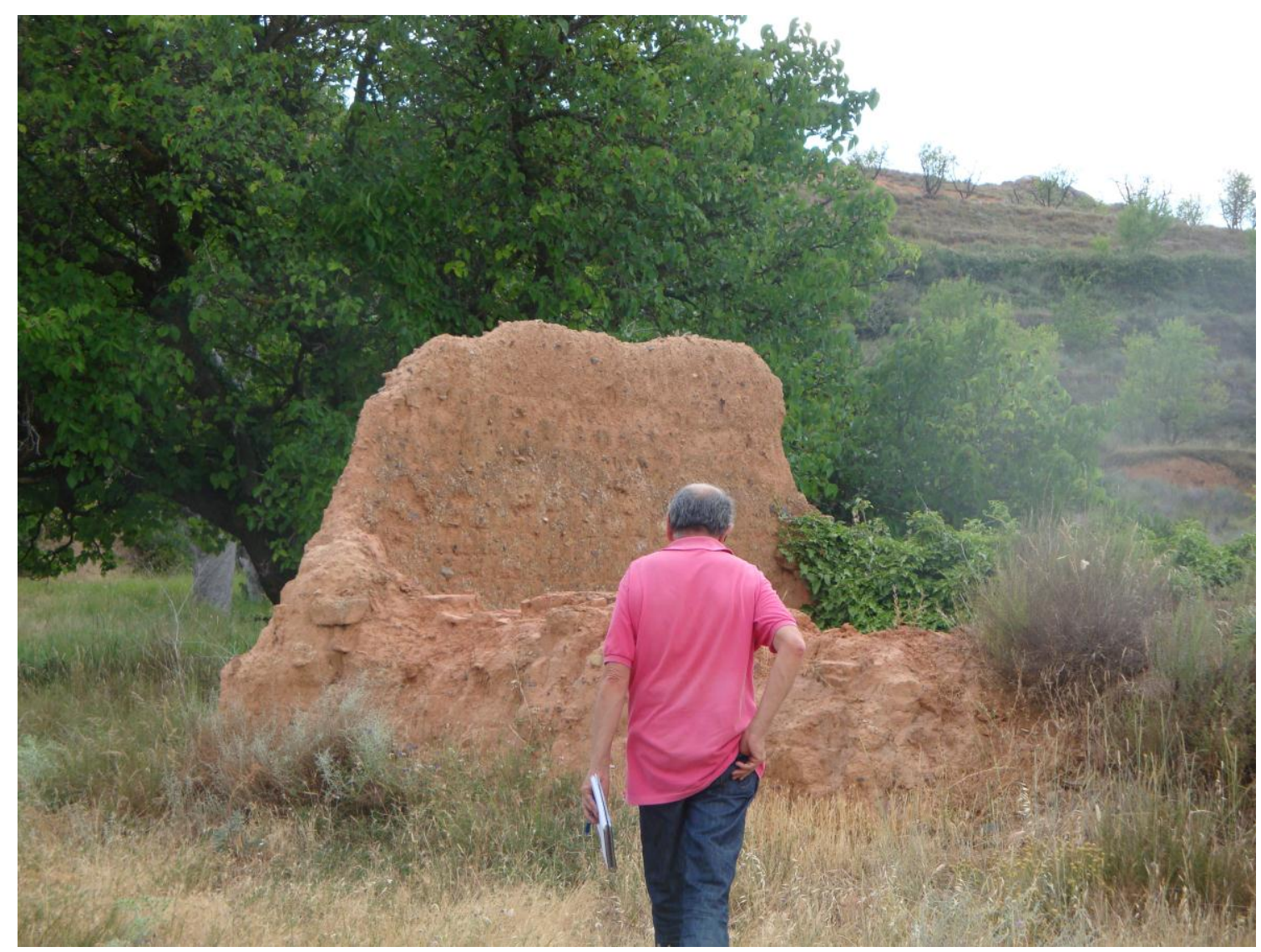

El Tejar de Santa Cruz de Moncayo

No obstante, conviene decir que dentro del pueblo se halla situado otro tejar, en un avanzado estado de deterioro.

PARADA 4. ARCILLERAS Y TEJAR DE LA UMBRIA ALTA NORTE, (término municipal de Tarazona, comarca del Moncayo). (Hoja $320)$.

Después de efectuar la parada anterior, conviene regresar hacía el cruce de carreteras. No obstante, poco después de la parada anterior, encontraremos un camino a la izquierda que se dirige hacía un conjunto de explotaciones de arcilla. Ahí, a menos de $0 ` 2 \mathrm{Km}$ de la anterior, efectuaremos una nueva parada.

En este recorrido, habremos encontrado afloramientos de los materiales lutíticos que predominas por todas partes. Así, en este sector se observan diversas explotaciones ahora cerradas.

En ese lugar, también se observa una vieja tejera, que forma parte de nuestro Patrimonio Minero. 


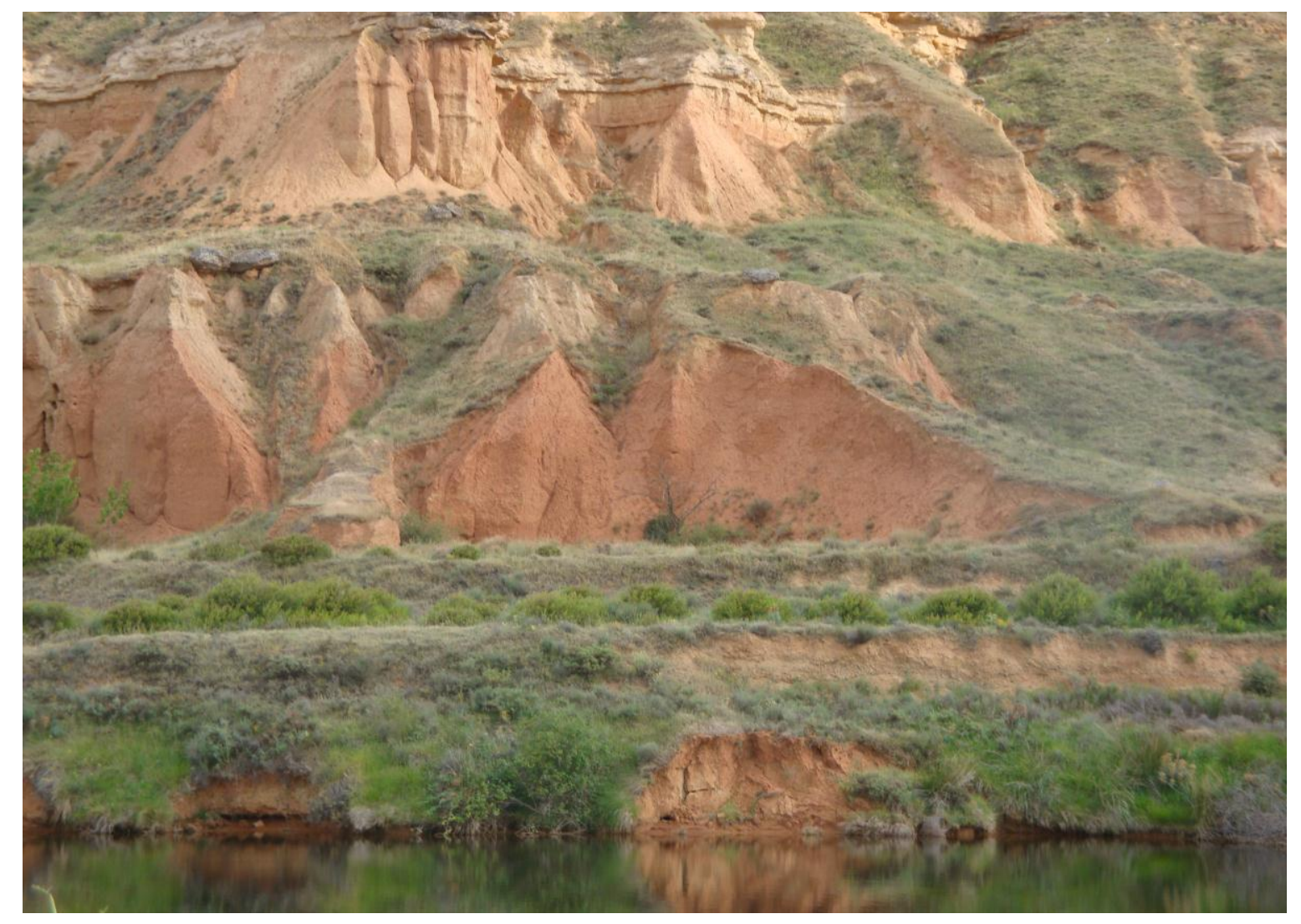

Afloramientos de calcolutitas ylutitas rojas.

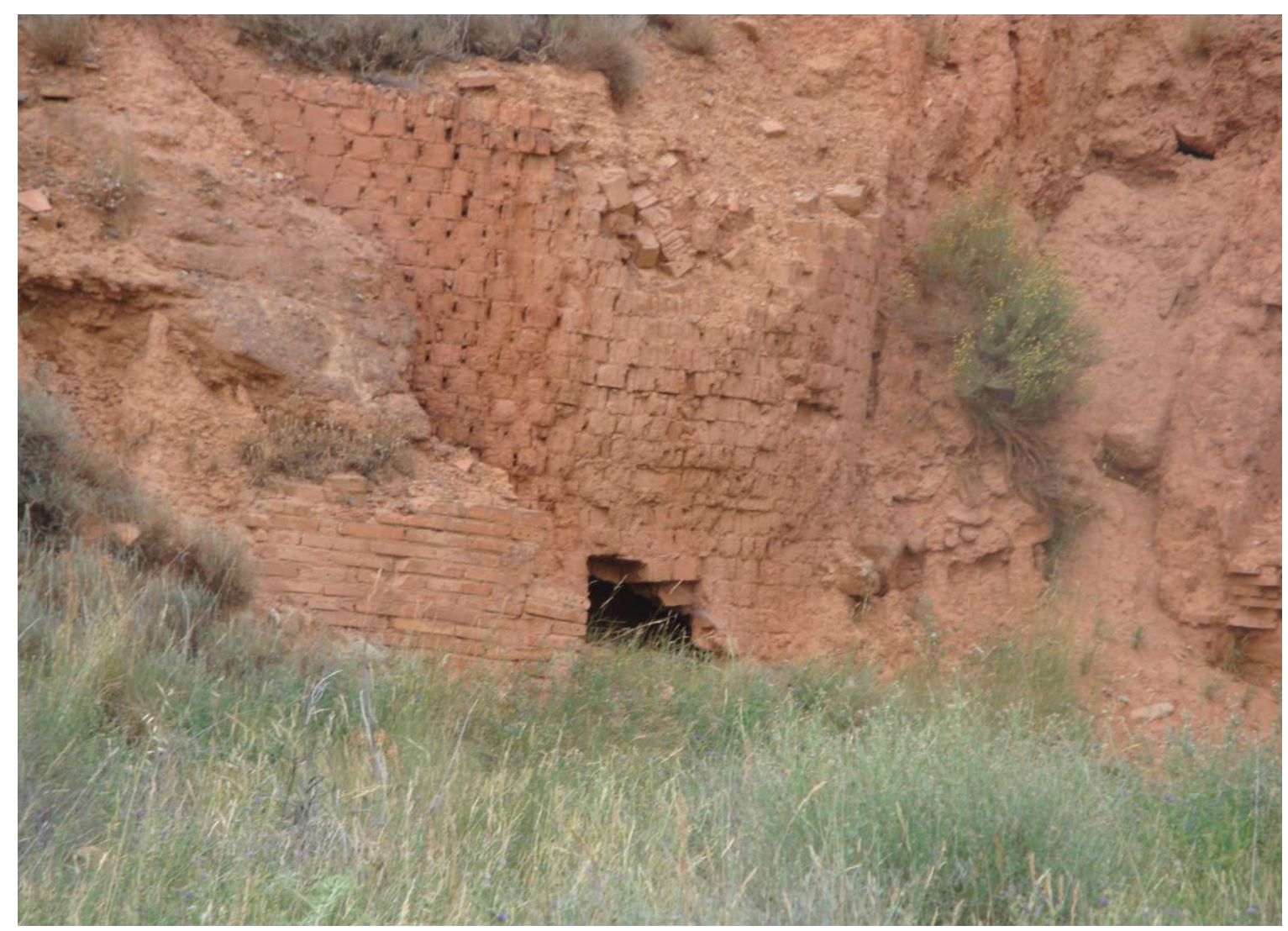

El Tejar 
PARADA 5. CANTERA DE LA UMBRIA ALTA - SUR, (término municipal de Tarazona, comarca del Moncayo). (Hoja 352).

Después de realizar la parada anterior, conviene continuar por la carretera que conduce al cruce de carreteras. Justo en el cruce nos conviene tomar (por la izquierda de la carretera) un camino que nos conducirá una antigua cantera de calcolutitas. Ahí efectuaremos una nueva parada, a unos $2 \mathrm{Km}$ de la anterior.

En este recorrido habremos ido encontrando los materiales citados en las paradas anteriores. No obstante, en este lugar hay un afloramiento de calcolutitas claras, pertenecientes al Mioceno.

Estos materiales han sido aquí explotados en una cantera, actualmente inactiva (como las anteriores canteras).

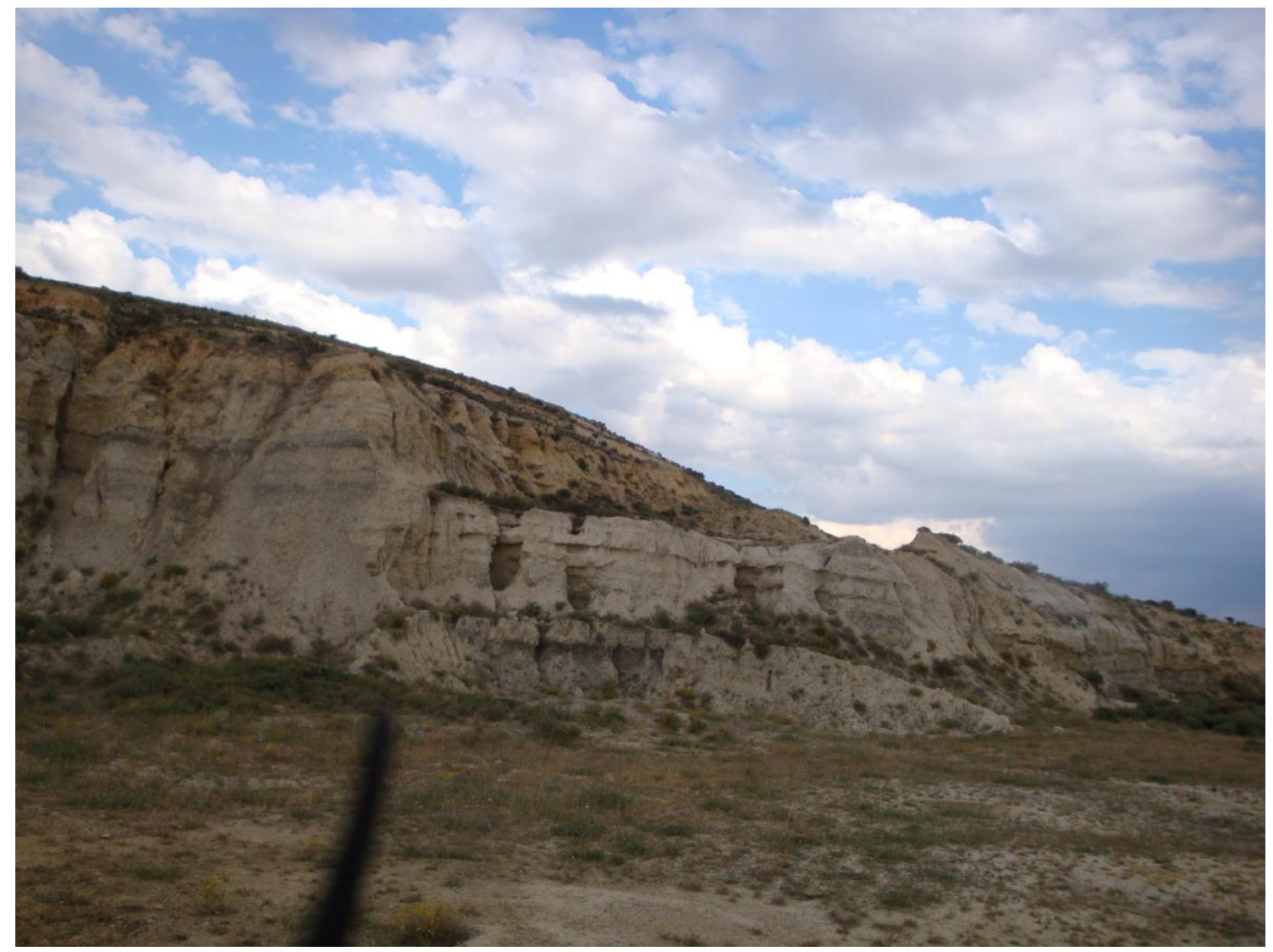

Cantera de la Umbría Alta (Sur)

PARADA 6. SUBIDA AL SANTUARIO DE LA VIRGEN DEL MONCAYO, (término municipal de Tarazona, comarca del Moncayo). (Hoja 352).

Tras realizar la parada anterior, cabe ahora continuar por la carretera, con la finalidad de ascender hasta el Santuario de la Virgen del Moncayo. Así, pronto se pasará 
por las inmediaciones de San Martín de la Virgen del Moncayo. Luego, se llegará a las inmediaciones de Agramonte, desde donde se continuará por la carretera (luego un camino) que conduce hasta el Santuario de la Virgen del Moncayo, donde llegaremos tras una larga subida. A unos $2 \mathrm{Km}$ antes de llegar efectuaremos una nueva parada, la última de este itinerario, a unos $15 \mathrm{Km}$ de la anterior

En este recorrido, al principio hemos pasado por una gravera situada al lado de la carretera, muy cerca del cruce. Luego, habremos encontrado afloramientos de los materiales cuaternarios de rellenos de piedemonte.

Finalmente, tras dejar la Depresión Geológica del Ebro, habremos entrado en el Sistema Ibérico (aunque los derrubios de pendiente nos habrán ocultado el contacto). A partir de ahí, hasta arriba, habremos encontrado los materiales paleozoicos que constituyen el Moncayo. Estos materiales del Carbonífero, son los que aparecen en el lugar de la parada.

En este lugar puede observarse un interesante canchal o colada de cascotes. Se trata de un conjunto de fragmentos de los materiales paleozoicos, triturados por fenómenos periglaciares. También podrían denominarse como ríos de piedras, en los cuales los fragmentos van avanzando y deslizándose sobre el hielo que se ha formado a partir del agua helada entre los fragmentos.

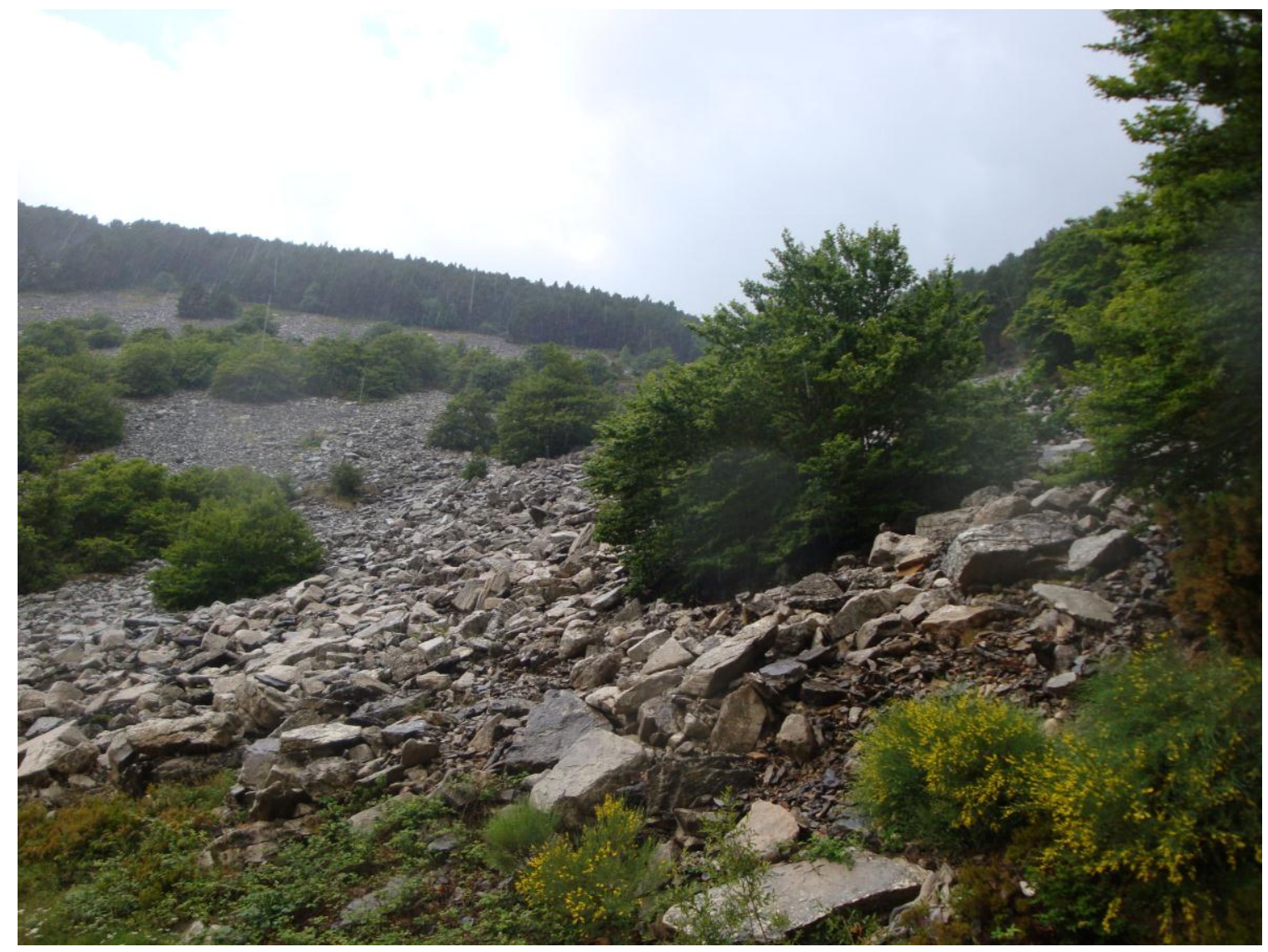

Un aspecto de los ríos de piedras

EN ESTE LUGAR FINALIZA EL ITINERARIO 


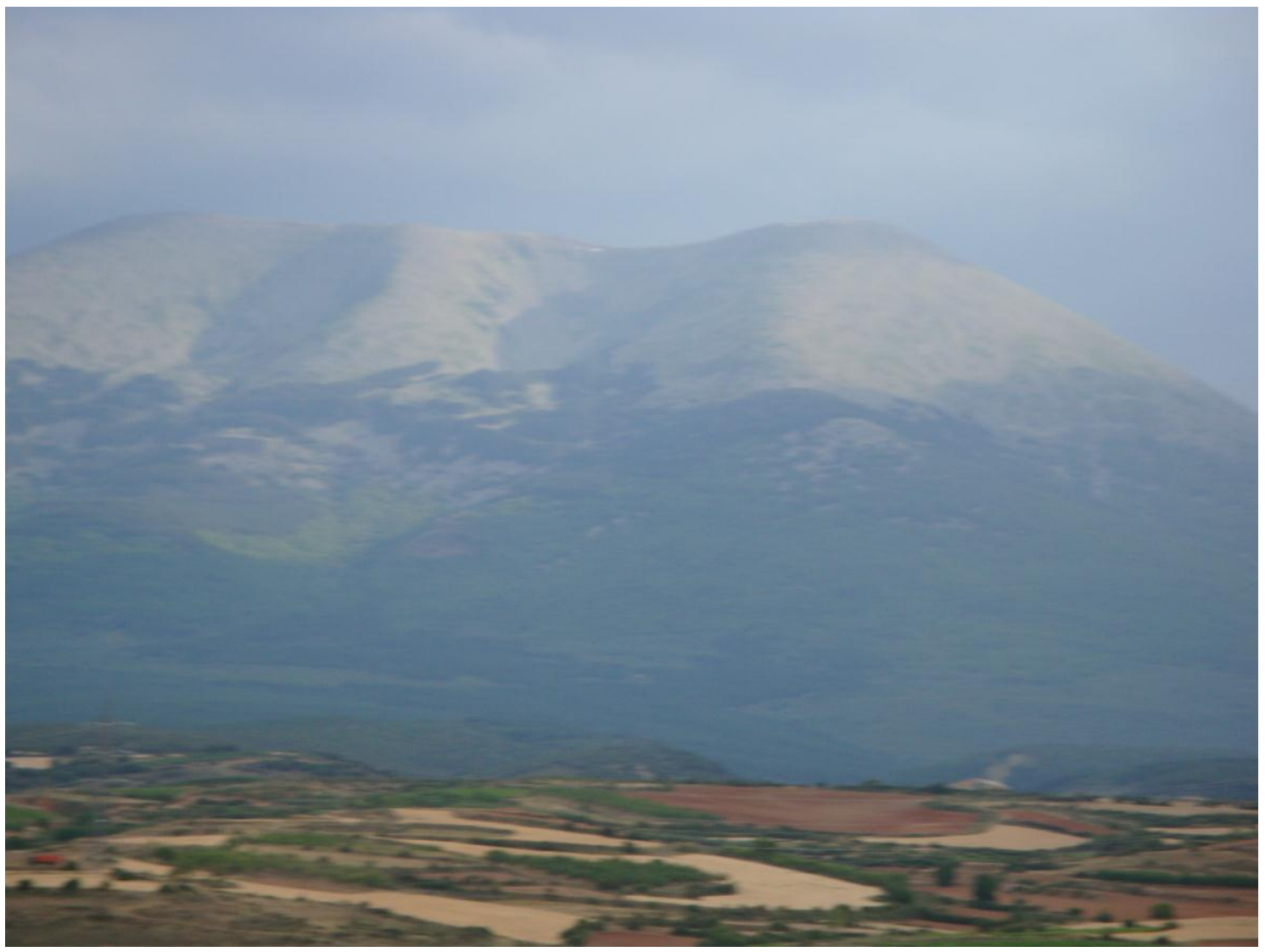

EL MONCAYO, DESDE LA UNBRIA ALTA

\section{BIBLIOGRAFÍA}

CALVO, M. (2001).- Las Minas de Sal de Remolinos, Zaragoza. Revista de Minerales, Vol 2, $n^{\circ} 2$, pp. 3 - 22. Barcelona

CALVO, M. et altri (1988). - Minerales de Aragón, Colección Temas Geológicos, 207 Pág. Zaragoza

GOBIERNO DE ARAGÓN (2001).- Puntos de Interés Geológico de Aragón. Consejería de Medio Ambiente del Gobierno de Aragón. Zaragoza

IGME (1972).- Mapa Geológico de España a escala 1:200.000 (Síntesis de la cartografía existente). Hoja y Memoria no ${ }^{\circ} 32$ (Zaragoza). Inst. Geol. Min. España

IGME (1973).- Mapa Metalogenético de España a escala 1:200.000. Hoja y Memoria nº 32 (Zaragoza). Inst. Geol. Min. España

IGME (1975).- Mapa de Rocas Industriales de España a escala 1:200.000. Hoja y Memoria $n^{\circ} .32$ (Zaragoza). Inst. Geol. Min. España 
MAESTRE, A. (1845).- Descripción geognóstica del Distrito Minero de Cataluña y Aragón. Anales de Minas, t. III. Madrid

MATA - PERELLÓ, J.; (1987).- Introducción al conocimiento de las mineralizaciones aragonesas. Mineralogistes de Catalunya, t.III, pp. 258-265. Barcelona

MATA - PERELLÓ, J.M. (2000).- Inventario Mineralógico de la comarca del Moncayo, Rodeno, 38. 24 Pág. Manresa

PRAMES (2005).- Moncayo. Colección RUTASCAL por Aragón. Prames, Gobierno de Aragón. 119 pag. Zaragoza. 